\title{
Not Just Numbers: Qualitative Research and the Clinical Neurosciences
}

Can J Neurol Sci. 2011; 38: 195-196

This issue of CJNS features a paper investigating patient perceptions of carpal tunnel syndrome (CTS) and ulnar neuropathy (UN) surgery. These disorders and the surgical interventions will be familiar to the readers of CJNS. The qualitative inquiry approach employed by the authors, Khu, Bernstein and Midha, ${ }^{1}$ however, with their accompanying data gathering methods and thematic analytic approach is likely novel for many CJNS readers.

Qualitative approaches are a mainstay of research in the social sciences and in education. Qualitative studies are also widely used in many health disciplines (e.g., medical education; nursing research), and they appear increasingly in core clinical journals such as NEJM, JAMA, Annals of Internal Medicine and BMJ. Qualitative inquiry can be a powerful tool for health researchers, one that stands on its own as a valid approach to understanding health phenomena as well as one that nicely complements traditional (e.g., quantitative, epidemiological) health research. ${ }^{2}$ Traditional quantitative and epidemiological research can be mobilized to answer important 'What?' and 'When?' questions; e.g., what are the predictors of a poor outcome with CTS surgery? When is surgical intervention indicated in patients with UN? Qualitative inquiry can further add to our knowledge by helping to understand 'How?' and 'Why?' questions. With their patients with CTS and UN, Khu and colleagues explore important non-quantifiable aspects of these conditions and their treatments, using probes like: "Did you perceive yourself as disabled?", "What made you decide to have surgery?", and "Have your expectations been met?" A combination of quantitative and qualitative approaches ("mixed methods" design) can be a particularly fruitful means to address multifaceted health phenomena.

We believe that many issues faced by patients and health care providers dealing with neurological and neurosurgical disorders are ideally suited to the tools of qualitative inquiry. However, a literature search in late 2010 using terms "qualitative" and "neurology" yielded barely a handful of studies. As Khu and colleagues remind us, good neurosurgical and neurological care encompasses a breadth of experience, skills and expertise. It is the responsibility of researchers and practitioners to canvass that breadth. While neurologists and neurosurgeons must base their practices on rigorous scientific evidence, they also need to attend to their patients' experiences: that is, the experiences of living with the symptoms and consequences of nervous system disorders, or the experiences of treatment and treatment outcomes. In the case of surgical procedures for CTS and UN, it is incumbent on the physician to understand both the disease and the illness: that is, the symptoms, signs, and pertinent investigations of these focal neuropathies, as well as the experience of the patient living with the disease, deciding to undergo nerve decompression, and then living with the consequences - good or bad - of the surgery.

Qualitative inquiry is not a monolith. It is an umbrella term that encompasses numerous approaches to health research. ${ }^{3} \mathrm{Khu}$ and colleagues have chosen an exploratory descriptive design using semi-structured interviews to better understand patient satisfaction with entrapment neuropathy surgery. Other data collection and analytic approaches for exploring issues relevant to such disorders and their treatments could also be employed. For example, grounded theory - a sociological methodology to understand social processes - could use face-to-face interviews and focus groups to understand the process of adapting to a condition such as CTS. Phenomenology - a philosophicallybased methodology to better understand human experience using in-depth interviewing could explore the everyday experience of living with a disability. Ethnography - an anthropological methodology to investigate socio-cultural phenomena - using participant observation could be employed to understand how our socio-cultural values and practices influence the provision of services for disabled members of our society.

Qualitative approaches can also be used in other complex areas of neurological health care. Kaptchuk and colleagues, ${ }^{4}$ for example, use a qualitative methodology to demonstrate how conventional theories of placebo inadequately attend to the myriad factors (physiological, embodied, social and cultural) that co-mingle to create what we call, in shorthand, the "placebo effect'. Similarly, Thorne and colleagues ${ }^{5}$ demonstrate with interpretive description the intricacies of good communication between health care providers and MS patients, and the iatrogenic effects of poor communication.

Critiques of qualitative inquiry as an approach to health research are often located in a debate about objective versus subjective engagement with data. In qualitative inquiry, the researchers themselves are the tools for the collection of data, given that they are embedded in the research contexts where they talk to and observe patients, health care providers and even policy makers. While subjectivity is indeed an important element of quality inquiry, this should not be taken to mean a lack of a rigorous research process. On the contrary, standards of rigor and critical appraisal are essential to qualitative inquiry, ensuring that research is conducted with the sophistication and precision one would expect from expert quantitative sciences. ${ }^{6}$ The careful development of the Cochrane Qualitative Research Methods Group, for example, is a case in point. Furthermore, quantitative and epidemiological research is not immune to subjectivity; Shrier and colleagues, for example, demonstrate how the interpretation of data in meta-analyses is a highly subjective process even among reviewers with extensive experience. ${ }^{7}$ 
We encourage readers who find their interests piqued by the potential of the qualitative approach to develop a habit of asking questions that are 'unanswerable' using traditional quantitative medical science. The practice of every neurologist and neurosurgeon is filled with such questions, and the application of qualitative methods to enhance our understanding of nervous system disorders has hardly begun.

Mary Ellen Macdonald, Colin Chalk

McGill University

Montreal, Quebec, Canada

\section{REFERENCES}

1. Khu KJ, Bernstein M, Midha R. Patients' perception of carpal tunnel and ulnar nerve decompression surgery. Can J Neuro Sci. 2011;38(2):268-73.

2. Pope C, Mays N. Qualitative research: reaching the parts other methods cannot reach: an introduction to qualitative methods in health and health services research. BMJ. 1995 Jul 1;311(6996): 42-5.

3. Kuper A, Reeves S, Levinson W. An introduction to reading and appraising qualitative research. BMJ. 2008;337:a288.

4. Kaptchuk T, Shaw J, Kerr C, et al. Maybe I made up the whole thing: placebos and patients experiences in a randomized controlled trial. Cult Med Psychiatry. 2009;33:382-411.

5. Thorne S, Con A, McGuinness L, McPherson G, Harris SR. Health care communication issues in multiple sclerosis: an interpretive description. Qual Health Res. 2004;14(1):5-22.

6. Kuper A, Lingard L, Levinson W. Critically appraising qualitative research. BMJ. 2008;337:a1035.

7. Shrier I, Boivin J-F, Platt R, et al. The interpretation of systematic reviews with meta-analyses: an objective or subjective process? BMC Med Inform Decis Mak. 2008;8(1):19. 